\title{
Universalisierung und Entpolitisierung von Klimawandelanpassung?
}

\section{Kritische Perspektiven auf die anwendungsorientierte Klimawandelanpassungsforschung}

\author{
Antje Bruns ${ }^{1} \mathbb{D} \cdot$ Hartmut Fünfgeld ${ }^{2}$ \\ Eingegangen: 31. März 2021 / Überarbeitet: 13. Mai 2021 / Angenommen: 20. Mai 2021 / Online publiziert: 23. Juni 2021 \\ (c) Der/die Autor(en) 2021
}

\section{Zusammenfassung}

In dem Maße, wie sich Klimawandelanpassung als Handlungsfeld von Kommunen, regionalen Gebietskörperschaften sowie Beratungs- und Planungsbüros verstetigt, findet eine zunehmende Standardisierung und Universalisierung von Konzepten, Ansätzen und Methoden statt. Diese Vereinheitlichung trägt zur Wissensintegration und -diffusion sowie zu einer weiteren Professionalisierung des Handlungsfeldes bei und bietet dadurch vermeintliche und tatsächliche Vorteile, z.B. bezüglich der Erprobung und Übertragbarkeit von Good Practices und im Bereich der Weiterbildung und des Kapazitätsaufbaus. Gleichzeitig birgt die Abkehr von einer prinzipiellen Vielfalt auf der Wissens- und der Handlungsebene auch Problematiken. Eine Orientierung an technologischen Innovationen, die möglicherweise zu neu entstehende Pfadabhängigkeiten führen und zugleich die Lern- und Veränderungsfähigkeit untergraben und den Blick auf notwendige Wertediskurse verstellen, können die Folge sein. In der Praxis wird Klimawandelanpassung häufig auf das Mach- und Umsetzbare reduziert, ohne z. B. Gerechtigkeitsfragen zu stellen. Vor dem Hintergrund dieser Beobachtungen beleuchten wir das Verhältnis von Wissensproduktion und Anpassungspraxis schlaglichtartig aus Perspektive einer kritischen Angewandten Geographie: (1) Epistemologien und Methoden sowie (2) theoretisch-konzeptuelle Ansätze (in) der Anpassungsforschung (3) eine deutliche Orientierung an Erfolgsgeschichten und (4) Prozesse der Wissensproduktion für eine konsensorientierte Politik der Klimaanpassung. Der Artikel möchte so einen Beitrag zur Auseinandersetzung mit aktuellen wissensbasierten Praktiken im Kontext des Handlungsfeldes Klimawandelanpassung leisten.

Schlüsselwörter Angewandte Geographie · Kritische Geographie · Wissenschaftliche Politikberatung · Anpassungswissen · Anpassungshandeln

Antje Bruns

brunsa@uni-trier.de

Hartmut Fünfgeld

hartmut.fuenfgeld@geographie.uni-freiburg.de
1 Fachbereich Raum- und Umweltwissenschaften, Universität Trier, Universitätsring 15, 54296 Trier, Deutschland

2 Institut für Umweltsozialwissenschaften und Geographie, Albert-Ludwigs-Universität Freiburg, Schreiberstr. 20, 79085 Freiburg i. Br., Deutschland 


\begin{abstract}
As climate change adaptation becomes more firmly established as a field of action for municipalities, regional authorities, as well as consulting and planning offices, increasing standardization and universalization of concepts, approaches, and methods are occurring. This standardization contributes to knowledge integration and diffusion as well as to further professionalization of the field of action, thus offering many potential and actual advantages, e.g., with regard to testing and reproducing good practice and in terms of further training and capacity building. At the same time, turning away from a theoretical plurality at the level of knowledge and action can be problematic too. Orientation toward technological innovations, possibly leading to newly emerging path dependencies, and at the same time undermining the ability to learn and change and obscuring the view of necessary value discourses, can be the consequence. In practice, climate change adaptation is often reduced to what is feasible and what can be implemented, without, for example, asking questions about justice. Against the background of these observations, we shed light on the relationship between knowledge production and adaptation practice from the perspective of critically applied geography: (1) epistemologies and methods; (2) theoretical-conceptual approaches to adaptation research; (3) a clear bias toward success stories; and (4) processes of knowledge production for a consensus-oriented climate adaptation policy. The article is thus aimed at contributing to the current knowledge-based practice in the context of the field of action of climate change adaptation.
\end{abstract}

Keywords Applied geography $\cdot$ Critical geography $\cdot$ Science-policy interface $\cdot$ Adaptation knowledge $\cdot$ Adaptation practice

\section{Einleitung}

[...] the issue of climate change, rather than being a problem of translating scientific matters of fact into political matters of concern, constitutes first and foremost, a political struggle over what to be concerned about (Goeminne 2012, S. 1).

Seit den 1990er-Jahren ist der Klimawandel ein wichtiges Forschungsthema der Geographie. Wurde der globale Klimawandel zunächst vorwiegend aus naturwissenschaftlicher Perspektive untersucht (Demeritt 2001), so bildete sich in den vergangenen Jahren eine sozialwissenschaftliche Anpassungsforschung heraus (Brunnengräber und Dietz 2013). Dieser Forschungsbereich betont, dass soziale, politische und ökonomische Verhältnisse nicht nur ursächlich für den Klimawandel sind, sondern auch den zentralen Ansatzpunkt für Anpassungskapazitäten, Handlungsmöglichkeiten und Veränderungsprozesse bilden. Unter anderem ist die sozialwissenschaftliche Anpassungsforschung an einem differenzierten Verständnis der durch Ungleichheiten gekennzeichneten sozialen Realitäten im Kontext des Klimawandels interessiert. Hingegen versteht sich dieser Forschungsbereich nicht als Hilfswissenschaft für die Übersetzung von (Klima-)Wissen in die (Anpassungs-)Praxis. Diese - oft von außen vollzogene - Zuschreibung wird von der sozialwissenschaftlichen Anpassungsforschung als reduktionistisch und simplifizierend abgelehnt, verweist aber auf die Notwendigkeit, das Verhältnis von Anpassungswissen und Anpassungspraxis zu beleuchten, und zwar gerade, weil „dem geographischen Erkenntnisstreben seit jeher weithin ein Praxisbezug innewohnt“ (Nuissl 2013, S. 19).
Basierend auf eigener Anpassungsforschung (vgl. Abb. 1), die dem Erwartungsanspruch der Anwendungsorientierung und Praxisrelevanz unterliegt, beobachten wir eine problematische Universalisierung und Entpolitisierung des Forschungs- und Handlungsfeldes Klimaanpassung. Dieser Trend wird durch eine Überbetonung von vermeintlichen Win-win-Lösungen und Erfolgsgeschichten, die Ungleichheiten eher überdecken als offenlegen, sowie durch eine weitgehend positivistische Rahmung des Klimawandels angetrieben. Vor diesem Hintergrund greifen wir mit diesem Beitrag aktuelle Kontroversen der Klimaanpassungsforschung auf und beleuchten diese aus

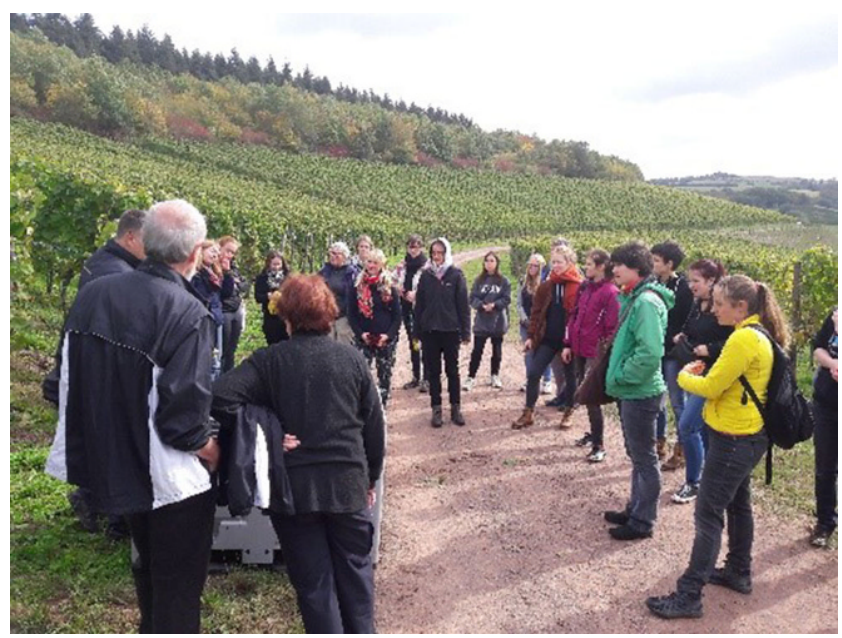

Abb. 1 Die Ko-Produktion von Klimaanpassungswissen spielt in den transdisziplinären Projekten Mosel-AdapTiV (Universität Trier) und LoKlim (Albert-Ludwigs-Universität Freiburg) eine zentrale Rolle. Beide Projekte wurden gemeinsam mit lokalen Akteuren entwickelt und dienen dem Auf- und Ausbau von Anpassungskompetenzen. (Foto aus dem Projektgebiet von Mosel-AdapTiv: Antje Bruns) 
Perspektive einer kritischen Angewandten Geographie (vgl. zum Verhältnis von Kritischer Geographie und Angewandte Geographie Kuge et al. 2020).

\section{Erkenntnistheoretische und methodische Grenzen}

Die Klima(wandel)forschung verfolgte zunächst das Ziel, einen gesicherten Nachweis zu erbringen, dass der Klimawandel tatsächlich stattfindet, anthropogen bedingt ist und sich auf dem globalen Maßstab vollzieht (Beck et al. 2013). Diese erste Ära der Klima(wandel)forschung fokussierte auf naturwissenschaftliche Phänomene und stützte sich auf quantitative Daten, die in standardisierte (Klima-)Modelle einflossen, um biophysikalische Wechselwirkungen zu verstehen. Diese Initialphase brachte den Nachweis des Klimawandels und begründete in der Folge die wissenschaftsbasierte Klimapolitik, wofür unter anderem die Gründung des Intergovernmental Panel on Climate Change (IPCC) im Jahr 1988 steht. Bis heute wurden 5 Klimasachstandsberichte veröffentlicht, die Wissen zusammentragen und für politische Entscheidungen aufbereiten (IPCC 2014). Eindrucksvoll dokumentieren diese Berichte den Zuwachs an Klimawissen; nicht zuletzt durch die Potenziale der Fernerkundung sowie durch die gestiegene Leistung von Klimarechenzentren sind die Modellierungen komplexer und integrativer geworden.

Nach wie vor besteht jedoch eine deutliche Schieflage zuungunsten sozialwissenschaftlicher Ansätze, ihren spezifischen Methoden und epistemologischen Annahmen (Nightingale 2016). Dafür ist auch die disziplinäre Arbeitsteilung verantwortlich, die 2 getrennte Forschungsfelder und Zuständigkeitsbereiche markiert. Während es üblicherweise im ersten Schritt darum geht, naturwissenschaftliche Grundlagenforschung zu betreiben, werden nachgelagert soziale, ökonomische und politische Fragen, einschließlich etwaiger Anpassungsmöglichkeiten, untersucht - und häufig sollen diese Forschungsbeiträge unmittelbare Lösungen vorschlagen. Durch diese Arbeitsteilung werden gesellschaftliche Verhältnisse als extern zum Klima(-wandel) konzeptualisiert (Goeminne 2012). Infolgedessen werden die heterogenen sozialen Realitäten, einschließlich ihrer inhärenten Ungleichheiten sowie kulturell unterschiedlicher Bewertungen des Klimawandels, weitgehend ausgeblendet (Adger et al. 2013). Diese Sozialblindheit hängt mit dem globalen Betrachtungsmaßstab zusammen, wie David Demeritt bereits vor 20 Jahren im Artikel ,The construction of global warming and the politics of science" darlegt. Die globale Rahmung des Klimawandels sei nämlich polit-ökonomischen Akteuren mit einem Interesse an der Zementierung des Status quo dienlich (ebd. 2001). Erst kürzlich bekräftigte ein Autor*innenkollektiv, dass die
Klima(wandel)forschung immer noch an Epistemen, Konzepten und Methoden festhalte, die der Transformation von Gesellschaft und Wissenschaft entgegenstehen (Nightingale et al. 2020). Aus Perspektive der kritischen Angewandten Geographie sind diese Argumente von Bedeutung, weisen sie doch darauf hin, dass die Rahmung und methodische Bearbeitung eines Themas (hier: Klimawandel) von unmittelbarer Relevanz für die Praxis (hier: Klimawandelanpassung) ist. Gerade integrativ-geographischen Arbeiten kommt die Verantwortung zu, durch eine vermeintliche Arbeitsteilung nicht zu einem verkürzten Problemverständnis (naturalisierend, entpolitisierend) beizutragen, auf dessen Basis schließlich nur bestimmte Anpassungsoptionen und -maßnahmen in den Blick geraten (so auch Beck et al. 2013).

\section{Positiv konnotierte theoretisch-konzeptuelle Ansätze}

In erkenntnistheoretischer Hinsicht lässt sich geographische Forschung zu Klimawandelanpassung in naturwissenschaftlich-positivistische Untersuchungen und in sozial- und geisteswissenschaftliche Arbeiten untergliedern. Als konzeptuelle Klammer haben sich - auch gerade in inter- und transdisziplinären Forschungskontexten - integrative Konzepte mittlerer Reichweite wie Vulnerabilität, Resilienz oder Anpassungskapazität etabliert (vgl. Meerow und Stults 2016). Diese nehmen meist eine eher funktionale Stellung als vage Grenzobjekte (engl. „,boundary objects“, Star und Griesemer 1989) ein, welche die Integration unterschiedlicher Perspektiven und Zugänge zum Forschungsgegenstand Klimawandelanpassung ermöglichen. In jüngster Zeit ist hierbei eine Verschiebung von einer ursprünglich problematisierenden Perspektive auf Klimawandelrisiken und Vulnerabilität hin zu positiv konnotierten Konzepten wie Resilienz (z.B. Davoudi et al. 2012) und - weniger ausgeprägt - Anpassungskapazität (z.B. Grothmann und Patt 2005) erkennbar. Solche Ansätze lassen die Gefahren und Risiken des Klimawandels trotz Komplexität und vielschichtiger Unsicherheiten als auf der individuellen oder kollektiven Ebene handhabbar erscheinen (Chandler 2014). Eine derartige semantische Verschiebung läuft jedoch Gefahr, die gesellschaftliche Brisanz sowie unabdingbare Werteabhängigkeiten von zusehends dringlichen politischen Entscheidungen im Handlungsfeld Klimawandelanpassung bewusst oder unbewusst auszublenden. Wenn damit der Blick von einer kritischen Auseinandersetzung mit Macht, (Un-)Gerechtigkeit und den politics von Klimawandelanpassung (vgl. Nightingale 2017) abgelenkt wird, können Grenzobjekte nicht länger als neutrale oder funktional-positive konzeptuelle Hilfsmittel verstanden werden (Brand und Jax 2007). Eine Blindheit gegenüber zutiefst wertebasierten, politisch kontrover- 
sen Entscheidungen auf Grundlage von positiv konnotierten Ansätzen kann neoliberal geprägte Klimaanpassungs-Gouvernmentalität legitimieren und verstetigen, bei der machtvolle Akteure eine kritische Auseinandersetzung mit den Anpassungsstrategien und -maßnahmen zu vermeiden und Verantwortung für die Anpassung auf Zielgruppen zu übertragen suchen (Joseph 2013). Wenn jedoch kritische Fragen zugelassen und eröffnet werden - beispielsweise im Rahmen von transdisziplinären Ansätzen - können im besten Fall wirksame Prozesse geschaffen werden, die vom Klimawandel besonders betroffene Bevölkerungsgruppen nicht nur einbeziehen, sondern sie auch dazu ermächtigen, Klimawandelanpassung(sforschung) aktiv mitzugestalten.

\section{Transdisziplinäre Orientierungen an Erfolgsnarrativen}

Nicht zuletzt aufgrund der entsprechenden Förderlinien auf Bundes- und Länderebene hat sich die Klimawandelanpassungsforschung in Deutschland in den letzten Jahren als transdisziplinäres Feld entwickelt, in dem im Rahmen von Reallaboren und anderen Formaten anwendungsorientiertes Wissen von Akteuren aus Wissenschaft und Praxis koproduziert wird. Bei transdisziplinären Ansätzen steht der praxisrelevante Erkenntnisgewinn durch reziprokes Lernen und die Möglichkeit des gemeinsamen Gestaltens von Anpassung im Vordergrund, während das rein wissenschaftliche Interesse an neuen Grundlagenerkenntnissen in den Hintergrund rückt. Die starke Fokussierung auf partizipative Lernprozesse in Anpassungsprojekten hat in den vergangenen Jahren viel zur Dokumentation von Good bzw. Best Practices und somit auch zur (Weiter-)Entwicklung von effektiven Ansätzen in der Klimawandelanpassung beigetragen. Damit einhergehend ist jedoch auch eine Eingrenzung von brauchbarem Wissen auf Erfolgsfaktoren und übertragbaren Fallstudien, während Fehlentscheidungen, Negativerfahrungen und das Scheitern von Maßnahmen nur selten dokumentiert werden und somit meist nur eingeschränkt in relevante Diskurse einfließen. Diese Form der Einhegung ist nicht zuletzt der auf Meilensteine ausgerichteten Förder- und Berichtslogik transdisziplinärer Projekte geschuldet, die vor dem Projektstart formulierte Ziele als Maßstab heranzieht. Gleichzeitig tragen Akteurs- und Projektnetzwerke zur selektiven Propagierung von Best Practices bei, sodass sich Anpassungsstrategien und -maßnahmen als mobile Politiken (McCann und Ward 2011) verbreiten und nachgeahmt werden. Best Practices und Erfolgsgeschichten verfügen daher über eine eigene, relationale Wirkmächtigkeit für evidenzbasierte Anpassungspolitik, die im besten Fall Inspiration für sekundäre Innovationen und Weiterentwicklungen liefert. Im schlimmsten Fall ruft sie jedoch technokratische Nachahmung, unreflektierte Standardisierung von Lösun- gen und nicht hinterfragte politische Legitimierung hervor (Webber 2015).

Eine intensive Auseinandersetzung mit der Aufarbeitung von Fehlern, Misserfolgen und dem Scheitern von Ansätzen kann dazu beitragen, Klimawandelanpassung als notwendigen Transformationsprozess zu verstehen, der eine prinzipielle Offenheit im Sinne eines zukunftsgerichteten Auslotens von radikal innovativen und flexiblen Maßnahmen erfordert. Geographische und sozialwissenschaftliche Untersuchungen auf der Diskurs- und Metaebene können aufzeigen, welche Interessengruppen neue (formelle und informelle) Leitbilder in welchen Sektoren und Handlungsbereichen vorantreiben, welche Ziele sie dabei verfolgen, und ob dabei Schließungsprozesse und Statuserhalt oder Öffnungen und Systemveränderung im Vordergrund stehen.

\section{Prozesse der Wissensproduktion für konsensorientierten Politikvorstellungen}

Die Angewandte Geographie befasst sich mit dem Verhältnis von Forschung und Praxis. Dieses Verhältnis hat sich im Bereich der Klima(wandel)forschung in den vergangenen Jahren deutlich gewandelt: Während Klimawissenschaftler zunehmend zu Dienstleistern werden, nehmen politische Entscheidungsträger vermehrt die Rolle von Kunden ein, die Sachexpertise einkaufen. Dies ist eine Reaktion darauf, dass Gebietskörperschaften nötiges Fach- und Erfahrungswissen im Umgang mit dem Klimawandel fehlt. Zukunftsorientiertes Klimaanpassungshandeln wird von vielen Akteuren erst allmählich mit kommunalen und regionalen Aufgaben, z. B. im Bereich der Daseinsvorsorge, in Verbindung gebracht. Dieses Missverhältnis trifft auf eine Forschungslandschaft, die erstens verstärkt auf Drittmittel angewiesen ist und zweitens Drittmittelgebern gegenübersteht, die anwendungs- und lösungsorientiertes Wissen einfordern. Aus dieser spezifischen Dynamik zwischen (Anpassungs-)Forschung und (Anpassungs-)Praxis resultiert ein besorgniserregender Trend, der zur Kommerzialisierung von Wissen und Entpolitisierung von Handlungsansätzen führt.

Beispielhaft für diesen Trend steht das Climate Service Center Germany (GERICS), das sich als „Ideenschmiede für Klimadienstleistungen“" versteht (www.climate-servicecenter.de) und nach einem Baukastenprinzip wissensbasierte Dienstleistungen anbietet (vgl. Abb. 2). Gegründet wurde es im Rahmen der bundesdeutschen Hightech-Strategie, mit der Innovationen für Wachstum, Wohlstand und Lebensqualität angesichts gegenwärtiger Herausforderungen hervorgebracht werden sollen. Als Element einer grünen Wachstumsstrategie werden also lösungs- und umsetzungsorientierte Klimadienstleistungen zu kommerziellen Produkten und zugleich werden wissenschaftliche Einrichtungen (GERICS gehört zum Helmholtz-Zentrum Geest- 
Abb. 2 Das Climate Service Center Germany (GERICS) bietet Klimaanpassungswissen im Baukastenprinzip an. (Quelle: https://www.climateservice-center.de/products_and_ publications/toolkits/index.php. de)

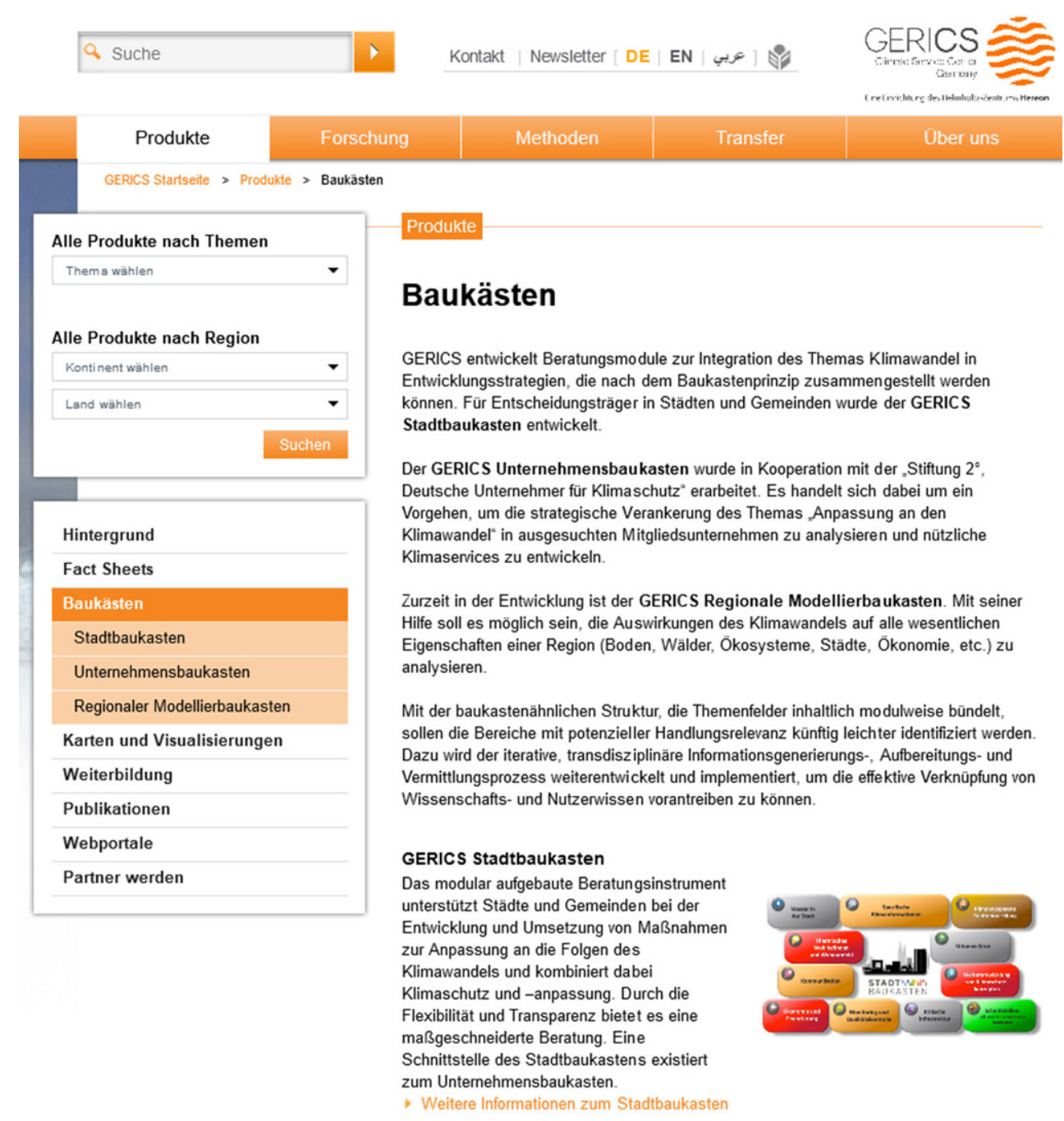

Sprachraum voranzubringen. Hierzu bestehen Anknüpfungsmöglichkeiten an internationale Diskurse, wovon wir einige in diesem Beitrag aufgegriffen haben. Die humangeographische Forschung hat in dieser Hinsicht viel zu bieten, vermag sie es doch, sich an der Schnittstelle zwischen Klima(natur)wissenschaft, Anwendungs- und Lösungsorientierung sowie fundierter Theoriediskurse Grenzbereiche aufzuzeigen und sich somit direkt und in gesellschaftsrelevanter Form mit „Klimawandel als Zustand“ (Bulkeley 2019) auseinanderzusetzen. Eine erste Zielvorstellung für Humangeograph*innen und andere Sozialwissenschaftler*innen könnte diesbezüglich sein, sich dem komplexen Machtgefüge, dem politischen Aushandlungsprozess und den damit verbundenen Fragen von sozialer (Un-)Gerechtigkeit von Klimawandel und Klimawandelanpassung mit radikaler Offenheit zu begegnen und hierzu in den vermehrten Austausch zu treten. In diesem Sinne hoffen wir, dass dieser Beitrag interessante Fragen für die Angewandte Geographie aufwirft. 
Funding Open Access funding enabled and organized by Projekt DEAL.

Open Access Dieser Artikel wird unter der Creative Commons Namensnennung 4.0 International Lizenz veröffentlicht, welche die Nutzung, Vervielfältigung, Bearbeitung, Verbreitung und Wiedergabe in jeglichem Medium und Format erlaubt, sofern Sie den/die ursprünglichen Autor(en) und die Quelle ordnungsgemäß nennen, einen Link zur Creative Commons Lizenz beifügen und angeben, ob Änderungen vorgenommen wurden.

Die in diesem Artikel enthaltenen Bilder und sonstiges Drittmaterial unterliegen ebenfalls der genannten Creative Commons Lizenz, sofern sich aus der Abbildungslegende nichts anderes ergibt. Sofern das betreffende Material nicht unter der genannten Creative Commons Lizenz steht und die betreffende Handlung nicht nach gesetzlichen Vorschriften erlaubt ist, ist für die oben aufgeführten Weiterverwendungen des Materials die Einwilligung des jeweiligen Rechteinhabers einzuholen.

Weitere Details zur Lizenz entnehmen Sie bitte der Lizenzinformation auf http://creativecommons.org/licenses/by/4.0/deed.de.

\section{Literatur}

Adger WN, Barnett J, Brown K, Marshall N, O’Brien K (2013) Cultural dimensions of climate change impacts and adaptation. Nature Clim Change 3:112-117

Beck S, Böschen S, Kropp C, Voss M (2013) Jenseits des Anpassungsmanagements. $\mathrm{Zu}$ den Potenzialen sozialwissenschaftlicher Klimawandelforschung. Gaia 22:8-13

Brand FS, Jax K (2007) Focusing the meaning(s) of resilience: resilience as a descriptive concept and a boundary object. Ecol Soc $12: 23$

Brunnengräber A, Dietz K (2013) Transformativ, politisch und normativ: für eine Re-Politisierung der Anpassungsforschung. Gaia 22:224-227

Bulkeley H (2019) Navigating climate's human geographies: exploring the whereabouts of climate politics. Dialogues Hum Geogr 9:3-17

Chandler D (2014) Beyond neoliberalism: resilience, the new art of governing complexity. Resilience 2:47-63

Davoudi S, Shaw K, Haider LJ, Quinlan AE, Peterson GD, Wilkinson C, Fünfgeld H, McEvoy D, Porter L (2012) Resilience: a bridging concept or a dead end? Plan Theory Pract 13:299-333

Demeritt D (2001) The construction of global warming and the politics of science. Ann Assoc Am Geogr 91:307-337

Goeminne G (2012) Lost in translation: climate denial and the return of the political. Glob Environ Polit 12:1-8. https://doi.org/10.1162/ GLEP_a_00104
Grothmann T, Patt A (2005) Adaptive capacity and human cognition: the process of individual adaptation to climate change. Glob Environ Chang 15:199-213

IPCC (2014) Climate change 2014: synthesis report. Contribution of working groups I, II and III to the fifth assessment report of the intergovernmental panel on climate change. IPCC, Genf

Joseph J (2013) Resilience as embedded neoliberalism: a governmentality approach. Resilience 1:38-52

Keele S (2019) Consultants and the business of climate services: implications of shifting from public to private science. Clim Change 157:9-26. https://doi.org/10.1007/s10584-019-02385-x

Kuge J, Naumann M, Nuissl H, Schipper S (2020) Angewandte und Kritische Geographie. Gemeinsame Herausforderungen, gemeinsame Perspektiven? Standort 44:219-225

McCann E, Ward K (Hrsg) (2011) Mobile urbanism: cities and policymaking in the global age. University of Minnesota Press, Minneapolis

Meerow S, Stults M (2016) Comparing conceptualizations of urban climate resilience in theory and practice. Sustainability 8:701

Nightingale AJ (2016) Adaptive scholarship and situated knowledges? Hybrid methodologies and plural epistemologies in climate change adaptation research. Area 48:41-47

Nightingale AJ (2017) Power and politics in climate change adaptation efforts: struggles over authority and recognition in the context of political instability. Geoforum 84:11-20

Nightingale AJ, Eriksen S, Taylor M, Forsyth T, Pelling M, Newsham A, Boyd E, Brown K, Harvey B, Jones L, Bezner Kerr R, Mehta L, Naess LO, Ockwell D, Scoones I, Tanner T, Whitfield S (2020) Beyond Technical Fixes: climate solutions and the great derangement. Clim Dev 12:343-352

Nuissl H (2013) Angewandte Geographie als anwendungsorientierte Grundlagenforschung. Standort 37:17-21

Star SL, Griesemer JR (1989) Institutional ecology, 'translations' and boundary objects: amateurs and professionals in Berkeley's museum of vertebrate zoology, 1907-39. Soc Stud Sci 19:387-420

Swyngedouw E (2011) Depoliticized environments: the end of nature, climate change and the post-political condition. R Inst Philos Suppl 69:253-274

Webber S (2015) Mobile adaptation and sticky experiments: circulating best practices and lessons learned in climate change adaptation. Geogr Res 53:26-38

Antje Bruns Professorin für nachhaltige räumliche Entwicklung und Governance

Hartmut Fünfgeld Professur für Geographie des Globalen Wandels 